\title{
Innovative application of metal-organic frameworks for encapsulation and controlled release of allyl isothiocyanate
}

\author{
Elham Lashkari ${ }^{\mathrm{a}}$, Hao Wang ${ }^{\mathrm{b}}$, Linshu Liu ${ }^{\mathrm{c}}, \mathrm{Jing} \mathrm{Li}^{\mathrm{b}}$, Kit Yam ${ }^{* a}$ \\ ${ }^{a}$ Department of Food Science, Rutgers University, 65 Dudley Road, New Brunswick, \\ New Jersey 08901, USA. \\ ${ }^{\mathrm{b}}$ Department of Chemistry and Chemical Biology, Rutgers University, 610 Taylor Road, \\ Piscataway, New Jersey 08854, USA. \\ ${ }^{\mathrm{c}}$ Eastern Regional Research Center, Agricultural Research Service, US Department of \\ Agriculture, Wyndmoor, Pennsylvania 19038, USA.
}

\footnotetext{
* Corresponding author at: Department of Food Science, Rutgers University, 65 Dudley Road, New Brunswick, New Jersey 08901, USA. Tel.: +1 848-932-5467; E-mail address: kyam@ sebs.rutgers.edu E-mail addresses: elham.lashkari@ rutgers.edu; hao.wang@ rutgers.edu; Jingli@ chem.rutgers.edu; linshu.liu@ars.usda.gov; kyam@sebs.rutgers.edu
} 


\begin{abstract}
This research investigated the technical feasibility of metal-organic frameworks (MOFs) as novel delivery systems for encapsulation and controlled release of volatile allyl isothiocyanate (AITC) molecules. We hypothesized that water vapor molecules could act as an external stimulus to trigger the release of AITC molecules encapsulated in MOFs. To test this hypothesis, three MOFs-HKUST-1, MOF-74(Zn), and RPM6-Znwere selected based on their structural properties and AITC molecular characteristics. Results from adsorption-desorption and GC headspace analyses showed that these MOFs could encapsulate and retain AITC molecules within their pores under low (30-35\%) relative humidity $(\mathrm{RH})$ conditions. In contrast, the release of AITC molecules from all these MOFs was triggered under high RH (95-100\%) conditions. These findings along with results from SEM, TEM, and XRPD studies support our hypothesis that water vapors could trigger the AITC release from these MOFs, indicating that development of the AITC-MOFs delivering system is technically feasible.
\end{abstract}

Keywords: metal-organic framework (MOF), allyl isothiocyanate, encapsulation, controlled release, moisture-trigger, natural food preservative, and antimicrobial food packaging.

\title{
1. Introduction
}

A major challenge of the food industry is to reduce the conventional use of synthetic food preservatives and substitute them with their natural counterparts in food 
formulations. Natural preservatives are perceived to have lower toxicity, less negative environmental effects, and better consumer acceptance. For these reasons, the potential use of essential oils or their natural bioactive compounds as plant derived antimicrobial agents for preservation of various food products has received much interest recently (Tyagi and Malik, 2010; Philips and Laird, 2011; Hyldgaard, Mygind, and Meyer, 2012). Allyl isothiocyanate (AITC), a potent natural antimicrobial compound (Frankel et al., 2016), is derived mostly from plants such as black and brown mustard, horseradish, broccoli, and wasabi. It possesses outstanding antimicrobial characteristics against a broad spectrum of foodborne pathogens and food spoilage-inducing microorganisms especially in vapor phase even at low concentrations (Inouye, Goi, Miyauchi, Muraki, Ogihara, and Iwanami, 1983; Goi, Inouye, and Iwanami, 1985; Isshiki, Tokuoka, Mori, and Chiba, 1992; Delaquis and sholberg, 1997). AITC is typically added directly as a food-flavoring agent to meats, mustard and mayonnaise condiments, but there is also a good opportunity to add AITC as a natural preservative to improve food safety.

However, the direct addition of AITC to food products has two major limitations. First, AITC is unstable when added directly to the food product, as it may react or bind to some food components such as water, peptides, and amino acids that eventually may result in degradation or unavailability of all or part of added AITC. Consequently sufficient amounts of AITC no longer exist to act as an efficient natural food preservative (Chen and Ho, 1998; Nadarajah, Han, and Holley, 2005; Chacon, Buffo, and Holley, 2006). Second, its pungency when used at high concentrations is another limiting factor that hinders its direct incorporation into food matrices. To overcome the limitations of direct addition, this study proposes the use of metal-organic frameworks (MOFs) as novel 
delivery or carrier systems for encapsulation and controlled release of volatile AITC molecules. This novel antimicrobial packaging approach allows the successful implementation of AITC into food systems by improving its stability while eliminating or reducing its negative organoleptic impact on various food matrices (Plackett, GhanbariSiahkali, and Szente, 2007; Kim, Chung, Shin, Yam, and Chung, 2008; Park, Barton, and Pendelton, 2012; Dias, Soares, Borges, Sousa, Nunes, and Oliveira, 2013; Siahaan, Pendelton, Woo, and Chun, 2014; Kara et al., 2014; Otoni, Espitia, Avena-Bustillos, and McHugh, 2016).

The uniqueness of this approach is that the volatile AITC molecules can penetrate more easily into the bulk of food matrices, and its carrier (the MOFs particles) do not need to be in direct contact with the food. Metal-Organic Frameworks (MOFs) are a novel class of highly ordered crystals with 3D network structures. They are formed through self-assembly of metal ions or clusters - mostly transition metals such as $\mathrm{Cu}$, $\mathrm{Zn}$, and $\mathrm{Fe}$ - that are connected together by organic ligands as linkers. These frameworks are highly porous and possess remarkable inner surface areas (Huang et al., 2003; Mueller, Schubert, Teich, Puetter, Schierle-Arndt, and Pastre, 2006). Their inner channels or pores even can be tailored based on the desired functionality and characteristics of the guest molecules. These features make MOFs suitable candidates as delivery systems for encapsulation and controlled release of small vaporous bioactive compounds such as volatile AITC molecules. While MOFs has significant structural diversity and robust thermal and mechanical stability, their high affinity to water molecules can sometimes lead to structural breakdown (Rosi, Kin, Eddaoudi, Chen, O’Keeffe, and Yaghi, 2005; Küsgens, Rose, Senkovska, Fröde, Henschel, and Siegle, 2009; Vaughn et al., 2013). This 
behavior maybe used as a tool to induce release of entrapped AITC molecules from selected MOFs.

In this research, we hypothesized that the high amounts of relative humidity within the package of high moisture content food products such as fresh produce can act as an external trigger for the release of entrapped AITC molecules from selected MOFs in a timely manner. To our knowledge this study would be the first attempt to investigate the technical feasibility of these frameworks as novel AITC carriers for food safety and food industry applications.

\section{Materials \& Methods}

\subsection{Materials}

AITC (>98\% purity) was purchased from Matrix Scientific (Colombia, SC, USA). MOF-74(Zn) and RPM6-Zn [Zn $\left.{ }_{3}(\text { bpdc) })_{3}(\mathrm{apy})\right] \cdot 3.08 \mathrm{DMF} \quad($ bpdc = biphenyl-4,4'dicarboxylate, apy =4,4'-azobispyridine) microparticles were synthesized salvothermally in the Department of Chemistry and Chemical Biology, Rutgers University (Piscataway, NJ 08854) according to the methods published by Rowsell and Yaghi (2006) and Wang et al. (2016), respectively. HKUST-1 microparticles were purchased from Sigma-Aldrich (St. Louis, MO, USA). Pore characterization data and structural building components (types of metal and organic ligand) of the three MOFs are summarized in Table 1.

\subsection{Encapsulation and quantification of adsorbed AITC}

Adsorption-desorption study was conducted to assess the affinities and loading capacities of these selected MOFs as encapsulating agents for volatile AITC molecules. 
During the adsorption phase, nitrogen was used as the carrier gas for AITC molecules by flowing through a liquid AITC bubbler at $18^{\circ} \mathrm{C}$ and bringing AITC vapor into the cavities of these microparticles. Following adsorption was the desorption phase in which pure nitrogen was flowing through the sample at $30^{\circ} \mathrm{C}$ so that AITC molecules loosely bound or adsorbed on the MOF surface rather than the inner cavities were washed away until a constant weight obtained. Sample weights were monitored and recorded during both phases of the experiment using a TA Instrument Q50 thermogravimetric analyzer (TGA).

\subsection{GC operating condition}

Headspace analysis of AITC was conducted using a 5890A Hewlett Packed gas chromatograph equipped with a DB-1 capillary column $(30 \mathrm{~m}$ long, $0.32 \mathrm{~mm}$ inner diameter, $1 \mu \mathrm{m}$ film thickness) and a flame ionization detector. The inlet and flame ionization detector temperatures were set at 220 and $250^{\circ} \mathrm{C}$, respectively. Column temperature started at $50^{\circ} \mathrm{C}$, held for 3 minutes, and then ramped from 50 to $180^{\circ} \mathrm{C}$ at a rate of $10^{\circ} \mathrm{C} / \mathrm{min}$. Helium was used as the carrier gas. The area under the curve for each concentration was obtained from the chromatograph software. Seven concentrations 2, 4, $10,20,40,64,100 \mathrm{ppm}$ (as $\mu \mathrm{L}$ AITC/L air) of AITC (98\% purity) were used to prepare the standard calibration curve. Each concentration was measured in triplicate.

\subsection{AITC release measurements}

Release kinetics of encapsulated AITC from the MOF microporous particles was studied by conducting GC headspace analysis under both high $(95<\%$ RH $<100)$ and low $(30<\% \mathrm{RH}<35)$ relative humidity conditions at room temperature $\left(24 \pm 1^{\circ} \mathrm{C}\right)$. To produce and maintain high $\mathrm{RH}$ in $250 \mathrm{~mL}$ glass jars, $30 \mu \mathrm{L}$ water was injected to each glass jar using a clean gas tight $0.5 \mathrm{~mL}$ syringe (Baton, Rouge, LA, USA) through the airtight 
valve (Mininnert valves, Supelco, Bellefonte, PA, USA) at the onset of the experiment. For low RH no water was added. $0.5 \mathrm{~mL}$ headspace samples were taken at predetermined time intervals to measure AITC concentration changes inside the jars. Pure liquid AITC (non-encapsulated AITC) was added to a filter paper in $250 \mathrm{~mL}$ glass jar as the control. All experiments were conducted with at least two repetitions. The effects of various temperatures on release profile of volatile AITC molecules from RPM6-Zn microparticles was studied under high $\mathrm{RH}$ at 6,24 , and $35^{\circ} \mathrm{C}$. The temperature and relative humidity were monitored by using mini digital thermometer and hygrometer temperature humidity gauges (amazon.com) throughout the experiments.

\subsection{Morphological study of MOFs}

To investigate the effects of moisture on structural characteristics of these microporous particles, SEM and TEM studies were conducted on MOF microparticles exposed to both low and high RH. For SEM study, samples were mounted on stubs and sputter gold-coated by EMS 150R ES Sputter Coater (EM Sciences, Hatfield, PA) for 30 seconds. They were then observed under scanning electron microscopy, FEI Quanta 200F (Hillsboro, OR, USA) under an accelerating voltage of 5-10 KV in high vacuum mode. For TEM, Formvar resin (EM Science, Hatfield, PA) was coated on a microscope slide and floated onto a water bath. Acetone clean cupper grids (200 mesh) placed on the film and then it was collected using a parafilm. A small amount of powders was pressed onto the grids. The grids then were observed under a Philips transmission microscope (CM-12, Philips, Netherlands) with an accelerating voltage of $80 \mathrm{kV}$ and imaged by using a DVC detector controlled by AMT software (Danvers, MA, USA). 


\subsection{X-ray powder diffraction analysis (XRPD)}

Powder X-ray diffraction analysis was performed on MOF samples before and after their exposure to low and high RH conditions to better understand the mechanism of AITC release from each MOF and the effect of moisture on AITC release from each MOF. MOF microparticles were finely distributed on a glassy lamella, and a Rigaku Ultima-IV X-ray diffractometer using $\mathrm{Cu} \mathrm{K} \alpha$ radiation $(\lambda=1.5406 \AA)$ was used to record the diffracted patterns of each sample. Graphite monochromator was used and the generator power settings were at $40 \mathrm{kV}$ and $40 \mathrm{~mA}$. Data were collected between a $2 \theta$ of $3-50^{\circ}$ with a step size of $0.02^{\circ}$ at a scanning speed of $2.0 \mathrm{deg} / \mathrm{min}$.

\section{Results \& Discussion}

\subsection{MOFs Characteristics}

MOF selection for encapsulation and controlled release of AITC molecules was based on two main factors: MOFs' pore size and their affinity or structural vulnerability to moisture. First, an efficient encapsulation requires the encapsulating agent to be able to efficiently entrap and store the adsorbate molecules within its pores; as larger pores will lead to immediate release of the guest molecules, while pore sizes smaller than the size of the guest molecules will not allow absorption to occur. Second, MOFs' structural vulnerability or affinity to moisture will allow the moisture to act as the external stimulus to trigger the release of encapsulated AITC molecules in a timely manner when the relative humidity in the headspace is high. As a result, HKUST-1, MOF-74(Zn), and RPM6-Zn were selected as AITC storage and delivery agents based on their structural 
properties including pore size and their affinity or sensitivity to water molecules. The structural characteristics and schematic views of these MOFs are shown in Table 1 and Figure 2, respectively.

\subsection{Adsorption-desorption analysis of AITC}

The adsorption-desorption study was conducted to evaluate the potential application of these MOFs as carriers for encapsulation of AITC molecules. The adsorption-desorption profiles of AITC from these MOFs are shown in Figure 1. The adsorption phase shows the loading or the mass of AITC adsorbed by the MOFs, and the desorption phase shows the mass of AITC retained by the MOF after nitrogen flushing. The small decline in mass during the desorption phase is due to the loss of some loosely adsorbed AITC molecules that were washed away by nitrogen. The loading capacities are 42, 27, and 14\% (wt AITC/wt MOF) for HKUST-1, RPM6-Zn, and MOF-74(Zn), respectively.

The higher uptake of AITC molecules by HKUST-1 and then RPM6-Zn microparticles can be attributed to their higher BET surface areas (shown in Table 1) and their distinctive structural features. The higher internal surface area of the MOF provides more affinity sites for adsorption of gas molecules, where gas molecules preferentially bind to surfaces in favor of interacting with other gaseous molecules. Therefore, MOFs with higher surface area absorb higher amount of the adsorbate molecules.

On the other hand, although the internal surface areas of MOF-74(Zn) and RPM6$\mathrm{Zn}$ microparticles are in a close range (between 496 and $609 \mathrm{~m}^{2} / \mathrm{gr}$ ), the uptake of AITC by RPM6-Zn microparticles (27\% wt AITC/wt MOF) is almost twice as much 
as MOF-74(Zn) microparticles (14\% wt AITC/wt MOF). This observation implies that factors other than the MOF surface area and pore volume affect the uptake of volatile AITC molecules by these MOFs. The cage-like pores of HKUST-1 particles and two-fold interpenetrating frameworks of RPM6-Zn particles were able to adsorb and retain higher amounts of AITC molecules within their inner cavities compared to the amounts of AITC adsorbed by the open channel framework of MOF-74(Zn) particles. In other words, the distinctive structural features of HKUST-1 and RPM6$\mathrm{Zn}$ strengthen the interactions between the guest molecules and the MOFs framework through enhancing entrapment and retention of volatile AITC molecules in close proximity of the framework atoms. This reasoning is also aligned with findings from Kesanli et al. (2005), Chun (2008), and Kuppler et al. (2009), indicating that MOFs with distinctive structural features such as cage-like pores and interpenetrating frameworks are superior candidates for sorption, storage and encapsulation of small gaseous molecules.

\subsection{Controlled release of AITC}

The efficacy of AITC encapsulation and later the release profile of AITC molecules by MOFs microparticles was studied under low (30-35\%) and high (95$100 \%$ ) relative humidity conditions at $24 \pm 1{ }^{\circ} \mathrm{C}$. As shown in Figure $3 \mathrm{a}$, the nonencapsulated AITC sample displays immediate release of AITC molecules compared to all encapsulated samples. All three MOFs are able to encapsulate and retain more than $90 \%$ of encapsulated AITC molecules within their pores at low RH. On the other hand, as Figure $3 \mathrm{~b}$ shows the release of AITC is triggered in the presence of high RH. These results support our hypothesis that the high relative humidity can trigger the release of 
AITC molecules from these MOFs. Yet, compared to non-encapsulated sample that immediate release occurred within the first few hours, the release of AITC molecules from all encapsulated trials showed an extended release profiles. An initial rapid release of up to $60 \%$ of encapsulated AITC that followed by a slower release period. At the end of the experiment $96,80,70 \%$ of encapsulated AITC molecules were released from MOF-74(Zn), HKUST-1, and RPM6-Zn, respectively.

Similar AITC release profiles were observed in studies conducted by Park, Barton, and Pendelton (2011), Park et al. (2012), and Siahaan et al. (2014) from mesoporous silica and brown seaweed Saccharina japonica microparticles. However, encapsulation in MOF microparticles has the advantage that release of AITC can be triggered by an external stimulus, which is exposure to high $\mathrm{RH}$, while the releases from both seaweed powder and mesoporous silica were passive and controlled by the pore size only. According to findings of Park et al. (2012), the early moderately high release rate of volatile AITC molecules from mesoporous silica within the first $10 \mathrm{hrs}$ of the release generated a rapid lethal dose of antimicrobial AITC against selected food-borne bacteria and fungi that successfully inhibited their growth. Besides, the extended slow release of AITC led to distinct reduction in viable counts of each pathogen. Also Inouye, Takizawa, and Yamaguchi (2001) reported that rapid evaporation of 14 essential oils when applied at sufficient concentrations is more effective than their slow evaporation to inhibit the growth of Streptococcus pathogenic bacteria.

While $96 \%$ of encapsulated AITC released from MOF-74(Zn), the observation that $70-80 \%$ of encapsulated AITC molecules in HKUST-1 and RPM6-Zn microparticles are released can be explained through the effects of two factors. First, 
moderate disruptions of 3D frameworks of RPM6-Zn particles by water molecules that substitute the organic ligands in coordination sites can hinder the release of entrapped AITC molecules. Second, formation of strong bonds between polar isothiocyanate end ($\mathrm{N}=\mathrm{C}=\mathrm{S}$ ) of AITC molecules and the available charged atoms of water clusters attached to the exposed coordination sites of RPM6-Zn and unsaturated metal centers (UMC) of HKUST-1 microparticles that may prevent some of the encapsulated AITC molecules from release. These water clusters when merge together can also hinder the release of some of the residual AITC molecules from the pores before all encapsulated AITC molecules can escape from the MOF cavities to the headspace.

It is worth mentioning that although MOF-74(Zn) releases the highest percentage (96\%) of the encapsulated AITC from its open pores, the amounts of AITC that released from this MOF was the lowest compared to the other two MOF due to its lower AITC loading capacity. Based on the loading capacities and percentage of release, the final amounts of AITC released from these three MOFs at high RH is in the order of HKUST1>RPM6-Zn>MOF-74(Zn).

The effect of temperature on AITC release from RPM6-Zn microparticles was also investigated at high RH. As shown in Figure 4, increasing temperature increases both rate and amounts of ATIC release from RPM6-Zn microparticles. At $6{ }^{\circ} \mathrm{C}$ that mimics the refrigerator temperature, $90 \%$ of the entrapped AITC molecules remained inside the pores, while almost all the entrapped AITC molecules were released from the MOF at $35^{\circ} \mathrm{C}$ in a very short period of time. A faster AITC release also was observed by Seo et al (2012) at $25{ }^{\circ} \mathrm{C}$ than at $4{ }^{\circ} \mathrm{C}$. We attributed these results to the effects of higher temperatures on increasing the diffusion rates and the reaction energy among the 
molecules that leads to the faster release of AITC molecules. Given that abusive high temperatures along with the presence of high $\mathrm{RH}$ will accelerate the microbial growth, the obtained results at 24 and $35^{\circ} \mathrm{C}$ will ensure the release of AITC soon enough and to sufficient quantity to the headspace of the food package to keep the packed food from developing any potential pathogenic or spoilage contamination during its shelf life.

\subsection{Morphological characteristics of MOFs}

SEM micrographs show that the morphology of samples kept at low RH remained almost intact with well-defined structures and smooth surfaces (Figures 5a, c, e). In contrast, samples kept at high RH show minor to major changes in their form and structure. HKUST-1 and MOF-74(Zn) show small cracks on surfaces, although they still maintain their overall integrity and forms (Figures 5b, d). TEM micrographs also show that the regular and well-defined outline of these two MOFs remain almost unchanged at both high and low RHs.

In contrast to HKUST-1 and MOF-74(Zn), RPM6-Zn went through major structural transformation when exposed to high $\mathrm{RH}$ (Figure 5f). Its rough and multilayer appearance is attributed to the transformation from a $3 \mathrm{D}$ framework to a nonporous structure by water molecules. Further evidence of this structural breakdown is provided by TEM micrographs that show regular and well-defined outline at low RH (Figure 6a) but irregular and rough outline with less dense structure at high RH (Figure 6b).

\subsection{Crystalline properties of MOFs}

Although the AITC release from RPM6-Zn can be explained by the structural breakdown observed by SEM and TEM imaging, the mechanism responsible for the AITC release from HKUST-1 and MOF-74(Zn) at high RH remains unknown. Hence 
XRPD analysis was conducted to determine whether breakdown occurred at the crystalline structure. Presence of crystalline structure at high RH means that although minor structural deformation in the form of small cracks occurred in these MOFs, water molecules did not cause the MOF crystalline structure to breakdown through organic ligands substitution at metal-ligand coordination sites in the two MOFs that leads to the AITC release.

Figure 7 shows that when exposed to low RH, X-ray patterns of the three MOFs were almost unchanged compared to those before $\mathrm{RH}$ exposure, indicating their highly ordered crystalline structures were maintained. When exposed to high $\mathrm{RH}$, the X-ray patterns of HKUST-1 and MOF-74(Zn) were still unchanged, but the X-ray pattern of RPM6-Zn was changed substantially indicating significant loss of its highly order structure. These results along with findings from SEM and TEM micrographs confirmed that AITC release from RPM6-Zn is driven by transformation of a 3D network structure to a nonporous structure. As discussed in Wang et al. (2016), the substitution of organic ligands mainly $4,4^{\prime}$ 'azobispyridine pillar ligands by water molecules at metal-ligand coordination sites $(\mathrm{Zn}-\mathrm{N})$ leads to the MOF structural breakdown and release of entrapped AITC molecules.

Based on the results from SEM, TEM, and XRPD analyses, we propose that the replacement of entrapped less-polar AITC molecules by polar water vapor molecules within the highly polar pores and channels of HKUST-1 and MOF-74(Zn) is the driving force causing the AITC release from the two MOFs. As shown in Figure 2a and b, the large hydrophilic pores and unsaturated metal centers consist of $\mathrm{Cu}_{2}(\mathrm{OOC}-)$ paddlewheel secondary building unit (SBU) in HKUST-1, and hydroxyl groups of organic ligands in 
MOF-74(Zn) open channels are the hydrophilic attraction sites for water molecules. The adsorbed water molecules act as the main affinity sites for adsorption of other water molecules through hydrogen bonding, which ultimately result in the formation of small water clusters that force the exit of AITC molecules from the pores and channels of the two MOFs. However, when these small water clusters grow larger and merge with neighboring water clusters quickly, they can in fact hinder the release of some of the residual AITC molecules from the pores before the full release of encapsulated AITC molecules occur. The results of X-ray powder diffraction also confirm that metal-O coordination sites in HKUST-1 and MOF-74(Zn) are more water stable than metal-N bonds in RPM6-Zn, making the two MOFs structurally more stable to high RH than the RPM6-Zn particles.

\section{Conclusion}

This research investigated the potential application of metal-organic frameworks as novel delivery systems for encapsulation and controlled release of volatile AITC antimicrobial molecules. The three MOFs were able to encapsulate AITC molecules with loadings ranging between 130 and $400 \mathrm{mg}$ AITC/gr MOF. Exposure to 30-35\% RH resulted in only $10 \%$ AITC release, with the remaining $90 \%$ still encapsulated inside the pores and channels of the MOFs. But exposure to $95-100 \%$ RH resulted in AITC release ranging between 70 and $96 \%$. This finding support our hypothesis that water vapor at high concentration can act as an effective trigger to release AITC molecules from these MOFs. 
SEM, TEM, and XRPD analyses show that exposure to low RH (30-35\%) did not cause structural changes in all three MOFs. While exposure to high RH (95-100\%) caused a major structural change in RPM6-Zn, it caused only minor structural changes in HKUST1 and MOF-74(Zn). This observation suggests that the major structural change caused by substitution of metal-nitrogen bonds at coordination sites with metal-oxygen(water) is the main factor affecting the AITC release from RPM6-Zn. However, the factor(s) affecting the AITC release from HKUST-1 and MOF-74( $\mathrm{Zn})$ are less obvious. This research propose that polar water vapor molecules replace less-polar entrapped AITC molecules entrapped inside the highly polar cavities of HKUST-1 and MOF-74(Zn) particles forcing the release of AITC molecules from the two MOFs.

This study provides encouraging results to indicate that the application of MOFs as novel delivery systems for encapsulation and controlled release of AITC molecules using high relative humidity as the external trigger is technically feasible. For commercial applications, these AITC-MOFs particles can be packed in sachets permeable to both AITC and water vapors, sealed and placed inside the food package (Otoni, Espitia, Avena-Bustillos, and McHugh, 2016). Upon the water vapor triggered release, volatile AITC molecules from the MOFs will diffuse to the food surfaces via the package headspace to inhibit microbial growth and extend shelf life. Designing the sachet with the proper dimensions and constructing it with a material with proper AITC and $\mathrm{H}_{2} \mathrm{O}$ permeability make a desired release rate achievable. Gonçalves, Pires, Soares, and Araújo (2009), Pires, Soares, Andrade, Silve, Camilloto, and Bernardes (2009), Soe, Bang, Kim, Beuchat, Cho, and Ryu (2012), and Otoni, Soares, Silva, Medeiros, and Junior (2014) also reported the development of antifungal and antibacterial AITC sachets for peanuts, 
cottage cheese, mozzarella cheese, and fresh spinach preservation suggesting the application of these AITC emitting sachets can be technically practical. We believe this research could make contribution to improve safety, storage, and transportation shelf life of fresh and high moisture content packaged foods.

\section{Acknowledgment}

The authors thank Mr. Uknalis from USDA-ARS-ERRC (Pennsylvania) for providing facility and assistance in obtaining SEM and TEM images for this study. HW and JL would like to acknowledge the partial support from Department of Energy, Basic Energy Sciences, division of Materials Sciences and Engineering through Grant No. DEFG02-08ER-46491 for the synthesis and structure characterization work.

\section{References}

Britt, D., Furukawa, H., Wang B., Glover, T., \& Yaghi, O. M. (2009). Highly efficient separation of carbon dioxide by a metal-organic framework replete with open metal sites. Proceedings of the National Academy of Sciences, 106(49), 20637-20640.

Chacon, P. A., Buffo, R. A., \& Holley, R. A. (2006). Inhibitory effects of microencapsulated allyl isothiocyanate (AITC) against Escherichia coli O157:H7 in refrigerated, nitrogen packed, finely chopped beef. International Journal of Food Microbiology, 107, 231-237. 
Chen, C., \& Ho, C. T. (1998). Thermal degradation of allyl isothiocyanate in aqueous solutions. Journal of Agriculture and Food Chemistry, 46, 220-223.

Chun, H. (2008). Low-level self-assembly of open framework based on three different polyhedra: metal-organic analogue of face-centered cubic dodecaboride. Journal of American Chemical Society, 130, 800-801.

Delaquis, P. J., \& Sholberg, P.L. (1997). Antimicrobial activity of gaseous allyl isothiocyanate. Journal of food protection, 60 (8), 943-947.

Dias, M. V., Soares, N. F., Borges, S. V., Sousa, M. M., Nunes, C. A., \& Oliveira, I. N. (2013). Use of allyl isothiocyanate and carbon nanotubes in an antimicrobial film to package shredded, cooked chicken meat. Food Chemistry, 141, 3160-3166.

Frankel F., Priven. M., Richard E., Schweinshault C., Tongo O., Webster A., Barth E., Slejzer, K., \& Edelstein S. (2016). Health Functionality of Organosulfides: A Review. International Journal of Food Properties, 19, 537-548.

Glover, T. G., Peterson, G. W., Schindler, B. J., Britt, D., \& Yaghi, O. (2011). MOF-74 building unit has a direct impact on toxic gas adsorption. Chemical Engineering Science. $66,163-170$. 
Goi, H., Inouye, S., \& Iwanami, Y. (1985). Antifungal activity of powdery black mustard, powdery wasabi (Japanese horseradish), and allyl isothiocyanate by gaseous contact-Antifungal activity of plant volatiles. Journal of Antibacterial and Antifungal Agents, 13(5), 199-204.

Gonçalves, M. P. J. C., Pires, A. C. S., Soares, N. F. F., \& Araújo, E. A. (2009). Use of allyl isothiocyanate sachet to preserve cottage cheese. Journal of Food service, 20, 275279.

Huang, L. Wang, H., Chen, J., Wang, Z., Sun, J., Zhao, D., \& Yan, Y. (2003). Synthesis, morphology control, and properties of porous metal-organic coordination polymers. Microporous and Mesoporous Materials, 58, 105-114.

Hyldgaard, M., Mygind, T., \& Meyer, L. R. (2012). Essential oils in food preservation: mode of action, synergies, and interactions with food matrix components. Frontiers in Microbiology, 3, 1-23.

Inouye, S. Goi, H., Miyauchi, K., Muraki, S., Ogihara, M., \& Iwanami, Y. (1983). Inhibitory effect of volatile constituents of plants on the proliferation of bacteriaAntibacterial activity of plant volatiles. Journal of Antibacterial and Antifungal Agents, 11(1), 609-615. 
Inouye S., Takizawa T., \& Yamaguchi H. (2001). Antibacterial activity of essential oils and their major constituents against respiratory tract pathogens by gaseous contact. Journal Antimicrobial Chemotherapy, 47(5) 565-573.

Isshiki, K., Tokuoka, K., Mori, R., \& Chiba, S. (1992). Preliminary examination of allyl isothiocyanate vapor for food preservation. Bioscience Biotechnology and Biochemistry, 56(9), 1476- 1477.

Kara, H. H., Xiao, F., Sarkar, M., Jin, T., Sousa, M. M., Liu, C., Tomasula, P. M., \& Liu L. (2015). Antibacterial poly(lactic acid) (PLA) films grafted with electrospun PLA/allyl isothiocyanate fibers for food packaging. Journal of Applied Polymer Science. 4247542475.

Kesanli B., Cui Y., Smith, M. R., Bittner E. W., Bockrath B. C., Lin W. 2005. Highly interpenetrated metal-organic frameworks for hydrogen storage. Angewandte Chemie International Edition, 44, 72-75.

Kim, W. T., Chung, H., Shin, I. S., Yam, K. L., \& Chung, D. (2008). Characterization of calcium alginate and chitosan-treated calcium alginate gel beads entrapping allyl isothiocyanate. Carbohydrate Polymer, 71, 566-573 
Kuppler, R. J., Timmons, D. J., Fang, Q., Li, J., Makal, T. A., Young, M. D., Yuan, D., Zhao, D., Zhuang, W., \& Zhou, H. (2009). Potential applications of metal-organic frameworks. Coordination Chemistry Reviews, 253, 3042-3066.

Küsgens, P., Rose, M., Senkovska, I., Fröde, H., Henschel, A., \& Siegle, S. (2009). Characterization of metal-organic frameworks by water adsorption. Microporous and Mesoporous Materials, 120, 325-330.

Laird, K., \& Philips, C. (2011). Vapor phase: a potential future use for essential oils as antmicrobials? Letters in Applied Microbiology, 54, 169-174.

Moellmer, J., Moeller, A., Dreisbach, F., Glaeser, R., \& Staudt, R. (2011). High pressure adsorption of hydrogen, nitrogen, carbon dioxide and methane on the metal-organic framework HKUST-1. Microporous and Mesoporous Materials, 138, 140-148.

Mueller, U., Schubert, M., Teich, F., Puetter, H., Schierle-Arndt, K., \& Pastre, J. (2006). Metal-organic frameworks-prospective industrial applications. Journal of Material Chemistry, 16, 626-636.

Nadarajah, D., Han, J. H., \& Holley, R. A. (2005). Inactivation of Escherichia coli O157:H7 in packaged ground beef by allyl isothiocyanate. International Journal of Food Microbiology, 99, 269-279. 
Otoni, C. G., Soares, N. F. F., Silva, W. A., Medeiros, E. A. A., \& Baffa Junior, J. C. (2014). Use of allyl isothiocyanate-containing sachets to reduce Aspergillus flavus sporulation in peanuts. Packaging Technology and Science, 27, 549-558.

Otoni, C. G., Espitia P. J., Avena-Bustillos, R. J., McHugh T. H. (2016). Trends in antimicrobial food packaging systems: emitting sachets and absorbent pads. Food Research International, 83, 60-73.

Park, S. Y., Barton, M., \& Pendelton, P. (2011). Mesoporous silica as a natural antimicrobial carrier. Colloids and Surfaces, 385, 256-261.

Park, S. Y., Barton, M., \& Pendelton, P. (2012). Controlled release of allyl isothiocyanate for bacteria growth management. Food Control, 23, 478-484.

Pires, A. C. S., Soares, N. F. F., Andrade, N. J., Silva, L. H. M., Camilloto, G. P., \& Bernardes, P. C. (2009). Increased preservation of sliced mozzarella cheese by antimicrobial sachet incorporated with allyl isothiocyanate. Brazilian Journal of Microbiology, 40, 1002-1008.

Plackett, D., Ghanbari-Siahkali, A., \& Szente, L. (2007). Behavior of $\alpha$ - and $\beta$ cyclodextrin-encapsulated allyl isothiocyanate as slow-release additives in polylactic-copolycaprolactone films. Journal of Applied Polymer Science, 105, 2850-2857. 
Rosi, N. L., Kin, J., Eddaoudi, M., Chen, B., O’Keeffe, M., \& Yaghi, O. M. (2005). Rod packing and metal-organic frameworks constructed from rod-shaped secondary building units. Journal of American Chemical Society, 127, 1504-1518.

Rowsell, J. L., \& Yaghi, O. M. (2006). Effects of functionalization, catenation, and variation of the metal oxide and organic linking units on the low-pressure hydrogen adsorption properties of metal-organic frameworks. Journal of American Chemical Society, 128, 1304-1315.

Seo, H. -S., Bang, J., Kim, H., Beuchat, L. R., Cho, S. Y., \& Ryu, J. H. (2012). Development of an antimicrobial sachet containing encapsulated allyl isothiocyanate to inactivate Escherichia coli O157:H7 on spinach leaves. International Journal of Food Microbiology, 159, 136-143.

Siahaan, E. A., Pendelton, P., Woo, H., \& Chun, B. S. (2014). Brown seaweed (Saccharina japonica) as an edible natural delivery matrix for allyl isothiocyanate inhibiting food-borne bacteria. Food Chemistry, 152, 11-17.

Tyagi, A. K., \& Malik, A. (2010). Antimicrobial action of essential oil vapors and negative air ions against Pseudomonas florescence. International Journal of Food Microbiology, 143, 205-210. 
Vaughn, J., Wu H., Efremovska, B., Olsen, D., Mattai J., Ortiz, C., Puchalski, A., Li J., \& Pan, L. (2013). Encapsulated recyclable porous materials: an effective moisture-triggered fragrance release system. Chemical Communications, 49, 5724-5726.

Wang, H., Lashkari, E., Lim H., Zheng, C., Emge, T., Gong, Q., Yam, K., \& Li, J. (2016). The moisture-triggered controlled release of a natural food preservative from a microporous metal-organic framework. Chemical Communications, 52, 2129-2132. 

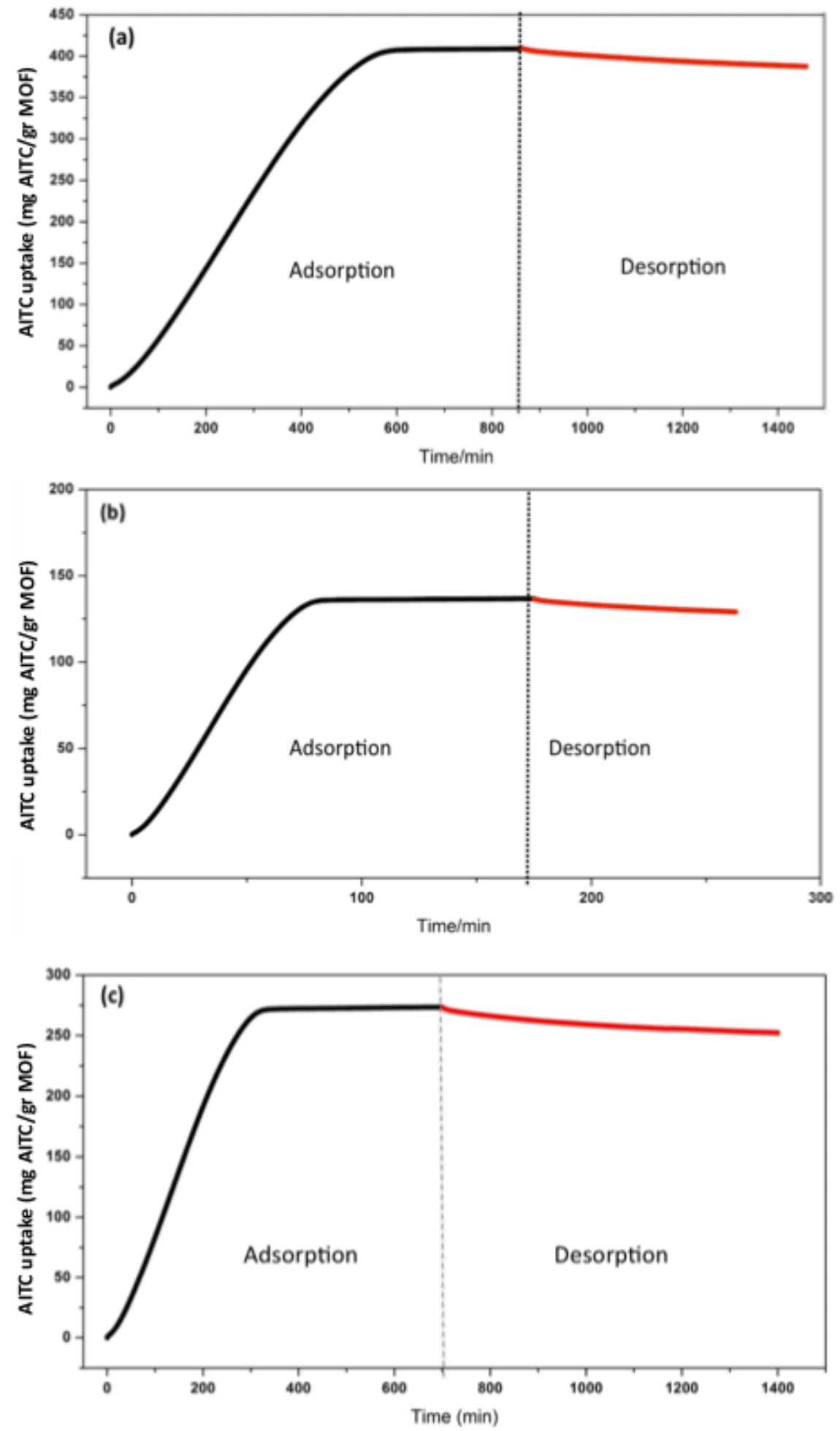

Fig 1. Adsorption-desorption profile of AITC on (a) HKUST-1, (b) MOF-74(Zn), and (c) RPM6-Zn microparticles. 


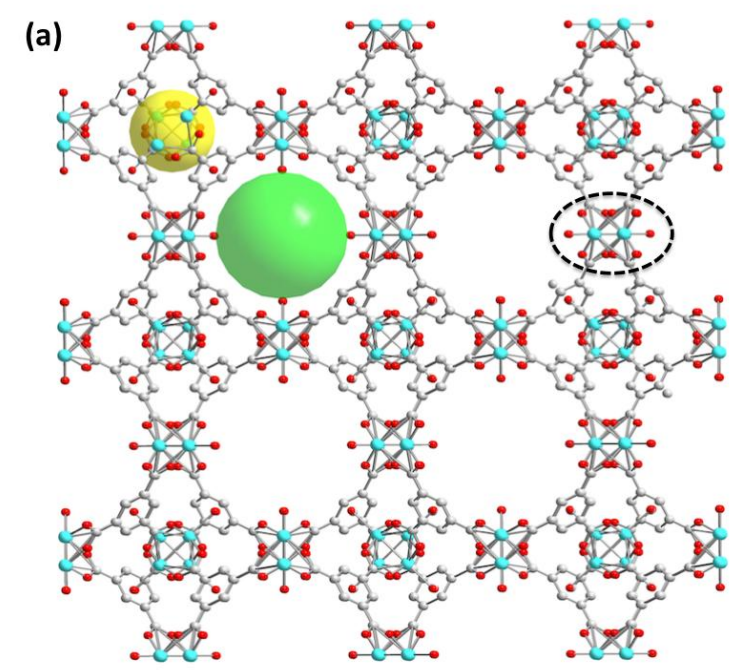

(b)
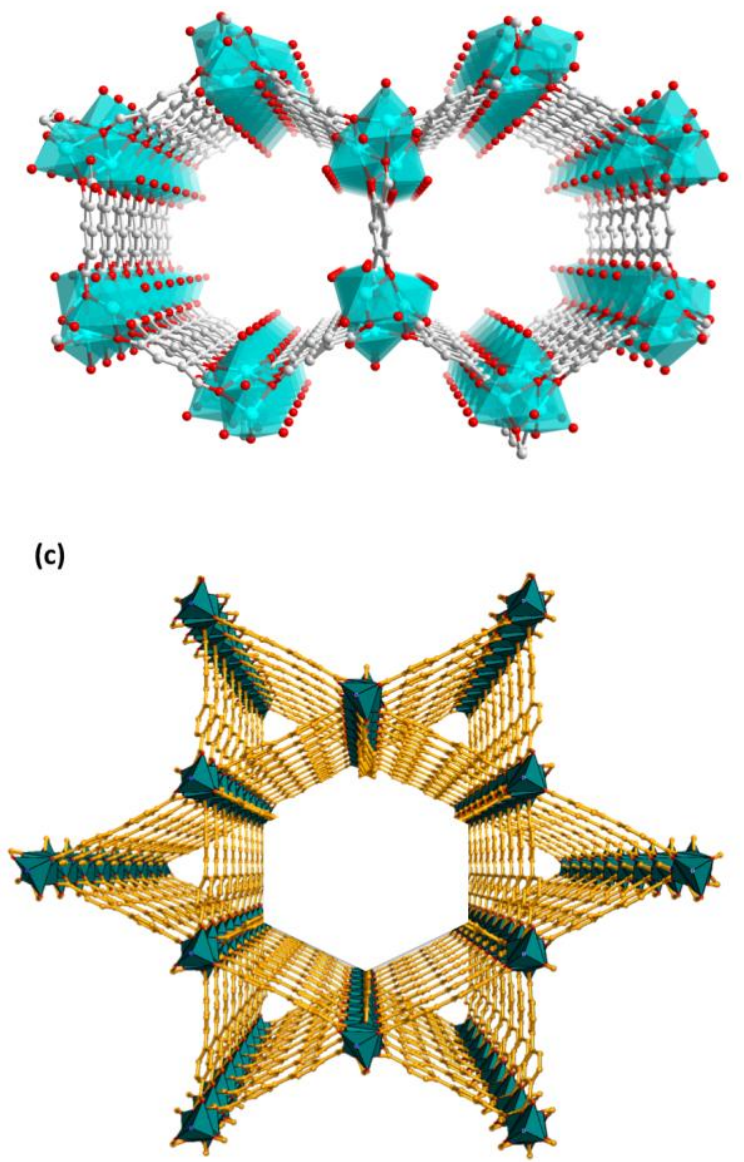

Fig 2. Chemical structure illustrations of (a) HKUST-1 in a two-dimensional view (teal:Cu, red:O, gray:C) with $\mathrm{Cu}$-paddlewheel SBU in black dashed circle and large and small pores shown in green and yellow spheres, (b) the honeycomb 1D open channels of MOF-74(Zn) framework; inorganic SBUs (teal) linked together via benzene rings of organic ligands (gray), (c) the 1D hexagonal channel of two-fold interpenetrated nets of RPM6-Zn; inorganic SBUs (green) linked together via organic ligands (yellow). 

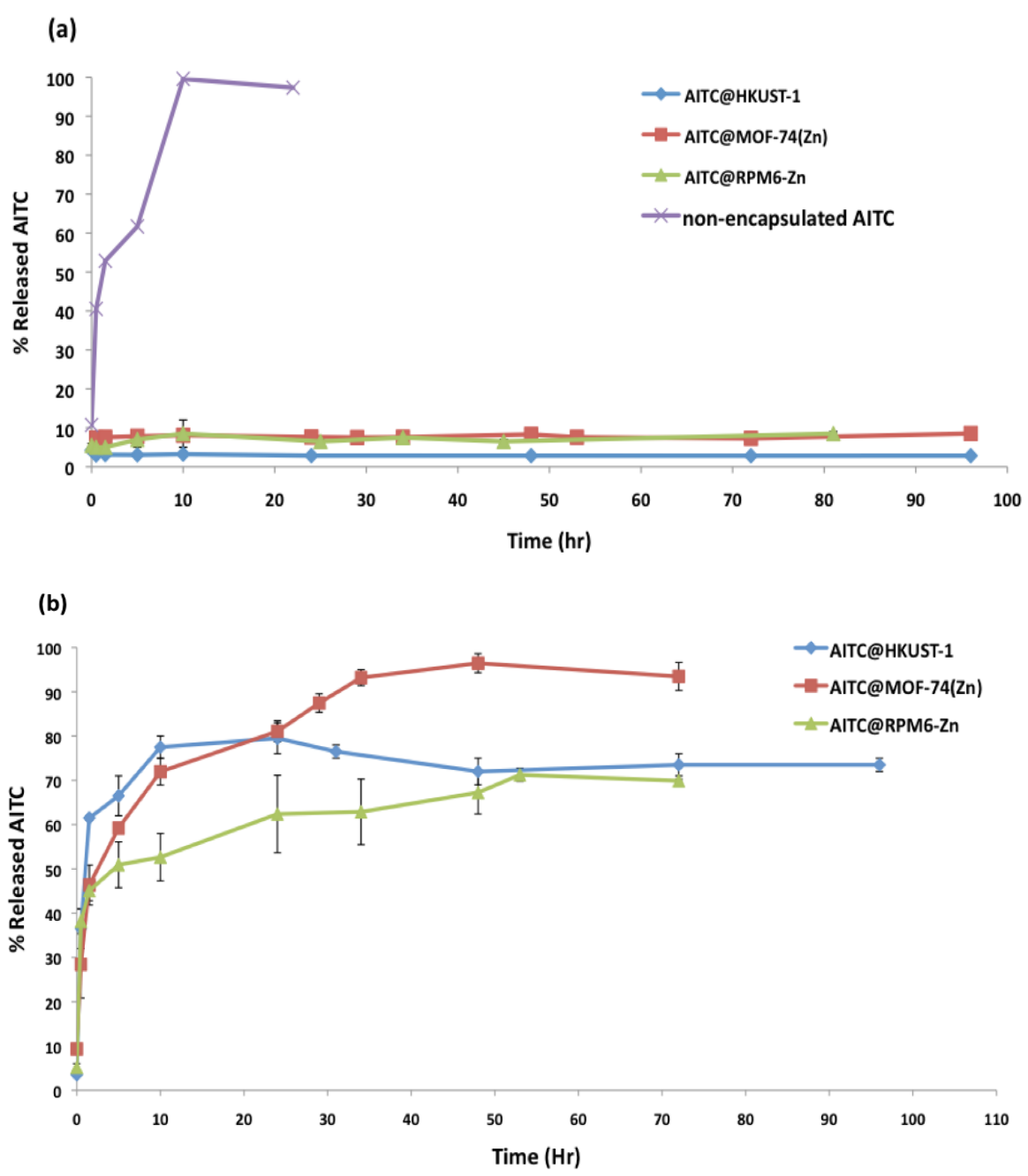

Figure 3. Release profile of AITC from three MOFs at (a) low (30-35\%) relative humidity, and (b) high $(95-100 \%)$ relative humidity conditions (standard error bars represent $\mathrm{STDV} / \mathrm{V}_{\mathrm{n}}$ ).

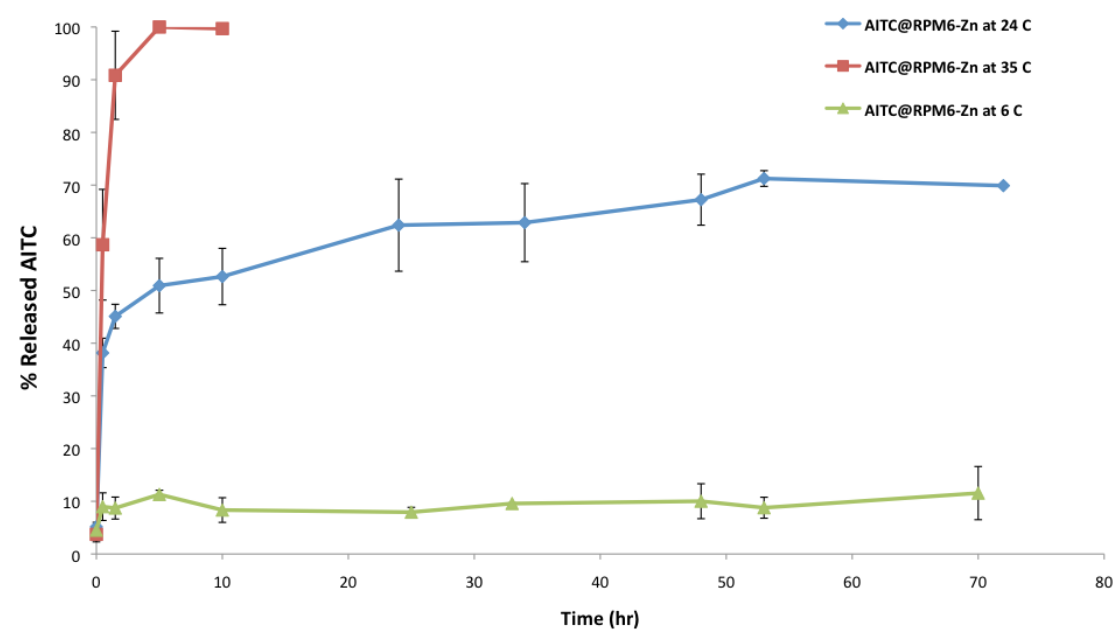

Figure 4. Release profile of AITC from RPM6-Zn particles at high (95-100 \%) relative humidity conditions and three different temperatures. 

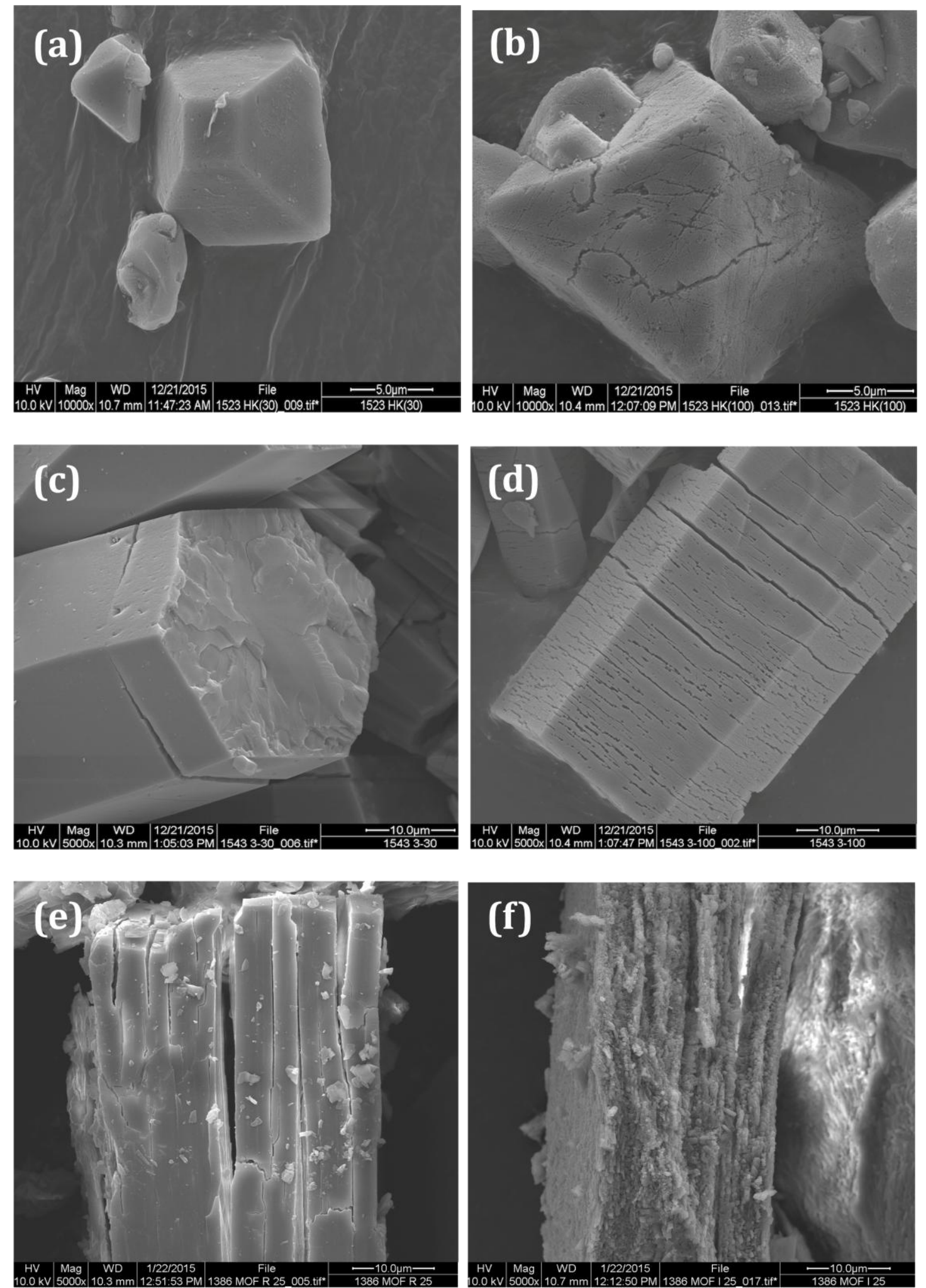

Figure 5. Scanning electron micrographs of (a) HKUST-1 particles kept at low RH; (b) HKUST-1 kept at high RH; (c) MOF-74(Zn)kept at low RH; (d) MOF-74(Zn) kept at high RH;(e) RPM6-Zn kept at low RH; (f) RPM6-Zn kept at high RH. High RH is $95-100 \%$ and low RH is $30-35 \%$. 


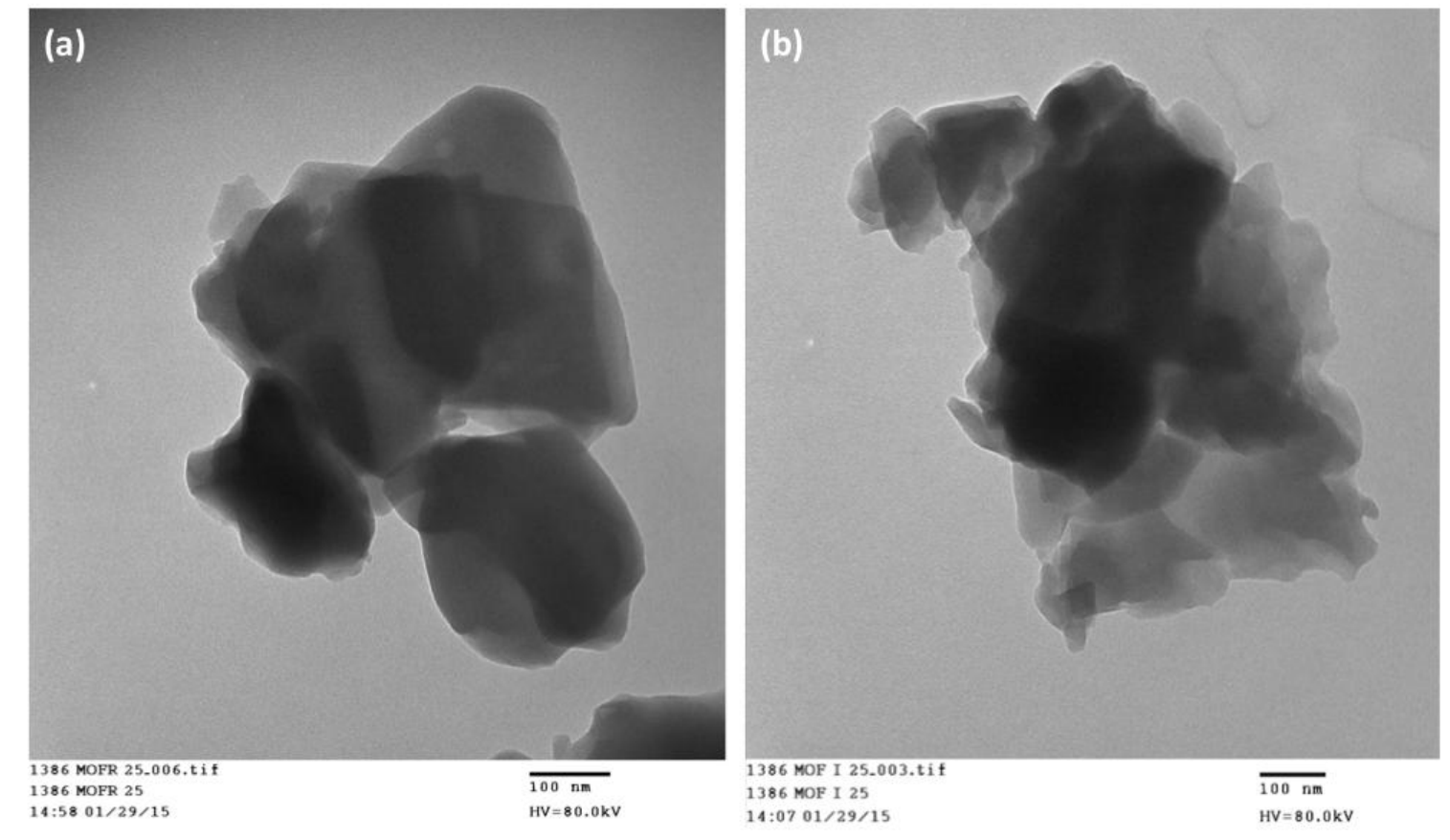

Figure 6. Transmission electron micrographs of (a) RPM6-Zn microparticles kept at low (30-35\%) RH, (b) RPM6-Zn microparticles kept at high (95-100\%) RH. 

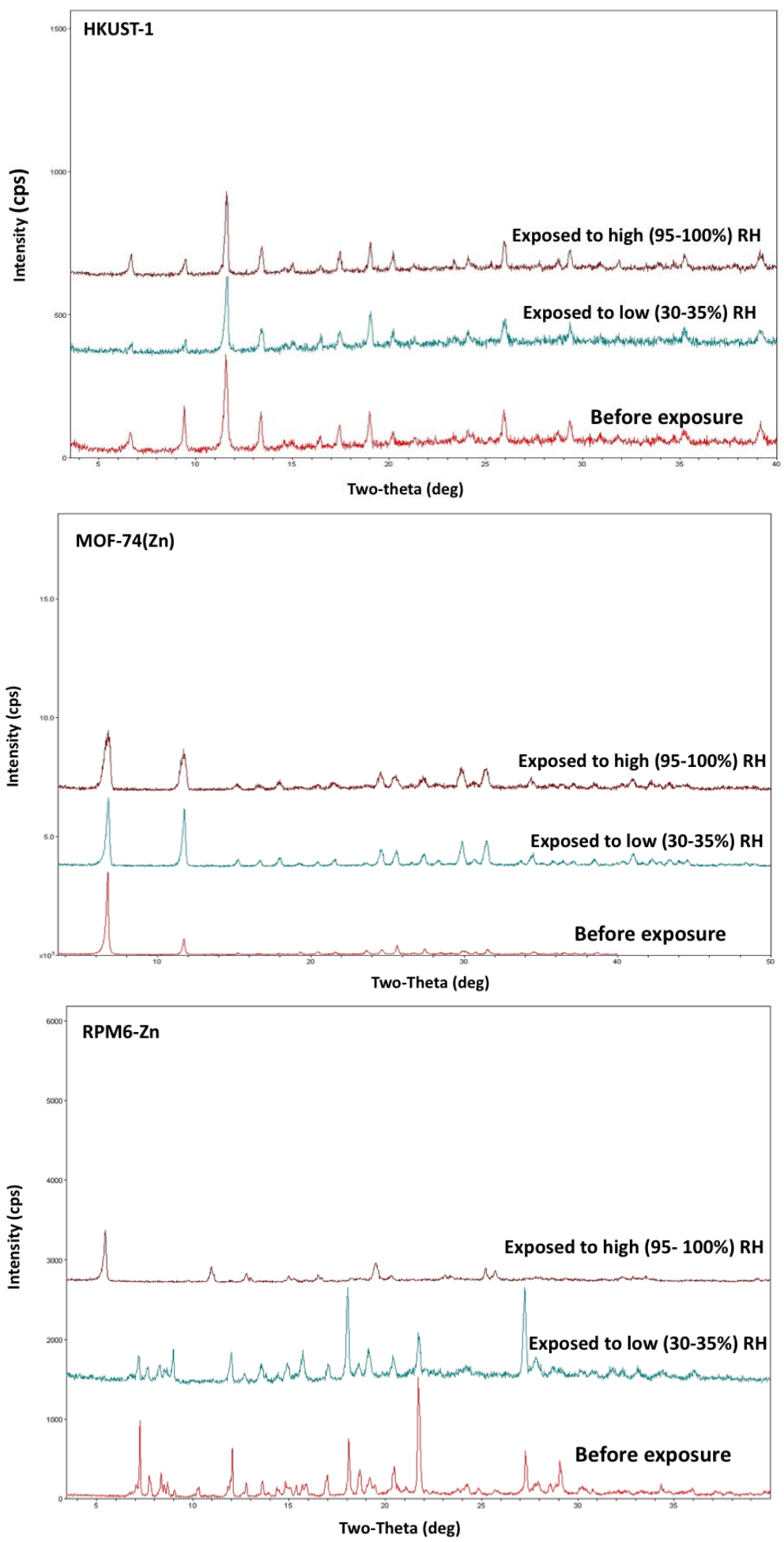

Figure 7. XRPD patterns of HKUST-1, MOF-74(Zn), and RPM6-Zn before RH exposure (red, bottom), after exposure to low (30-35\%) RH (green, middle), and after exposure to high (95-100\%) RH (brown, top). 
Table 1. Summary of MOFs pore characteristics and structural components

\begin{tabular}{|c|c|c|c|}
\hline & HKUST-1 & MOF-74(Zn) & RPM6-Zn \\
\hline $\begin{array}{l}\text { Organic } \\
\text { linker(s) }\end{array}$ & $\begin{array}{l}\text { 1,3,5-benzene } \\
\text { tricarboxylic acid }\end{array}$ & $\begin{array}{l}2,5- \\
\text { dihydroxyterephthalate }\end{array}$ & $\begin{array}{l}\text { Linking ligand: } \\
\text { biphenyl-4,4'-dicarboxylate } \\
\text { Pillar ligand: 4,4'- } \\
\text { azobispyridine }\end{array}$ \\
\hline Metal & Copper & Zinc & Zinc \\
\hline $\begin{array}{l}\text { Pore size } \\
(\AA))\end{array}$ & $\begin{array}{l}\text { Large pore: } 9 \\
\text { Small pore: } 6\end{array}$ & $5.5 \times 10$ & $7 \times 20$ \\
\hline $\begin{array}{l}\text { BET } \\
\text { surface } \\
\text { Area } \\
\left(\mathbf{m}^{2} / \mathrm{gr}\right)\end{array}$ & $1500-2100$ & 496 & 609 \\
\hline $\begin{array}{l}\text { Pore } \\
\text { Volume } \\
\text { (cc/gr) }\end{array}$ & 0.71 & NA & 0.29 \\
\hline References & $\begin{array}{l}\text { Küsgens et al., } 2009 \\
\text { Moellmer et al., } 2011\end{array}$ & $\begin{array}{l}\text { Britt et al., } 2009 \\
\text { Glover et al., } 2011\end{array}$ & Wang et al., 2016 \\
\hline
\end{tabular}

